\title{
SINGULAR JACOBI FORMS
}

\author{
JAE-HYUN YANG
}

\begin{abstract}
We introduce the differential operator $M_{g, h, h}$ characterizing singular Jacobi forms. We also characterize singular Jacobi forms by the weight of the associated rational representation of the general linear group. And we provide eigenfunctions of the differential operator $M_{g, h, M}$.
\end{abstract}

\section{INTRODUCTION}

Let $g$ and $h$ be two positive integers. Let $\mathscr{M}$ be a symmetric positive definite, half-integral matrix of degree $h$. For two positive integers $k$ and $l$, we denote by $\mathbf{R}^{(k, l)}$ the space of all $k \times l$ matrices with entries in the field $\mathbf{R}$ of real numbers. We let

$$
\mathscr{P}_{g}:=\left\{Y \in \mathbf{R}^{(g, g)} \mid Y={ }^{t} Y>0\right\}
$$

be the open convex cone of positive definite matrices of degree $g$ in the Euclidean space $\mathbf{R}^{g(g+1) / 2}$. We define the differential operator $M_{g, h, \mathscr{M}}$ on $\mathscr{P}_{g} \times$ $\mathbf{R}^{(h, g)}$ defined by

$$
M_{g, h, \mathscr{M}}:=\operatorname{det}(Y) \cdot \operatorname{det}\left(\frac{\partial}{\partial Y}+\frac{1}{8 \pi}^{t}\left(\frac{\partial}{\partial V}\right) \mathscr{M}^{-1}\left(\frac{\partial}{\partial V}\right)\right),
$$

where

$$
Y=\left(y_{\mu \nu}\right) \in \mathscr{P}_{g}, \quad V=\left(v_{k l}\right) \in \mathbf{R}^{(h, g)}, \quad \frac{\partial}{\partial Y}=\left(\frac{1+\delta_{\mu \nu}}{2} \frac{\partial}{\partial y_{\mu \nu}}\right)
$$

and

$$
\frac{\partial}{\partial V}=\left(\frac{\partial}{\partial v_{k l}}\right) \text {. }
$$

We note that this differential operator generalizes the differential operator $M_{g}$ $:=\operatorname{det}(Y) \cdot \operatorname{det}(\partial / \partial Y)$ on $\mathscr{P}_{g}$ which was introduced by $\mathrm{H}$. Maass (cf. [M]). Using the differential operator $M_{g}$, Maass (cf. [M], pp. 202-204) proved that if a nonzero singular modular form of degree $n$ and weight $k$ exists, then $n k \equiv 0(\bmod 2)$ and $0<2 k \leq n-1$. The converse was proved by $\mathrm{R}$. Weissauer (cf. [W], Satz 4).

Received by the editors June 8, 1994 and, in revised form, August 6, 1994

1991 Mathematics Subject Classification. Primary 11F30, 11F55.

This work was partially supported by KOSEF 901-0107-012-2, TGRC-KOSEF and the MaxPlanck-Institut für Mathematik. 
The aim of this paper is to characterize singular Jacobi forms. Singular Jacobi forms are defined to be the Jacobi forms which admit a Fourier expansion such that a Fourier coefficient $c(T, R)$ vanishes unless

$$
\operatorname{det}\left(\begin{array}{cc}
T & \frac{1}{2} R \\
\frac{1}{2} t R & \mathscr{M}
\end{array}\right)=0
$$

For more detail, we refer to Definition 2.2. This paper is organized as follows. In Section 2, we review the notion of singular Jacobi forms which was introduced by Ziegler (cf. [Z], Definition 3.7) and establish the notations. In Section 3 , we investigate some properties of the differential operator $M_{g, h, \mathscr{M}}$ to be used in the next section. In Section 4, we prove the main theorems. That is, we prove that singular Jacobi forms are characterized by $M_{g, h, \mathscr{M}}$ and the weight of the associated rational representation of the general linear group $G L(g, \mathbf{C})$. In the final section, we provide eigenfunctions of the above-mentioned differential operator $M_{g, h, M}$.

Notations. We denote by $\mathbf{Z}, \mathbf{R}$ and $\mathbf{C}$ the ring of integers, the field of real numbers, and the field of complex numbers respectively. $\operatorname{Sp}(g, \mathbf{R})$ denotes the symplectic group of degree $g . H_{g}$ denotes the Siegel upper half plane of degree $g$. For $M=\left(\begin{array}{ll}A & B \\ C & D\end{array}\right) \in \operatorname{Sp}(g, \mathbf{R})$ and $Z \in H_{g}$, we set $M\langle Z\rangle:=$ $(A Z+B)(C Z+D)^{-1} . \quad \Gamma_{g}:=\mathrm{Sp}(g, Z)$ denotes the Siegel modular group of degree $g .\left[\Gamma_{g}, k\right]$ (resp. $\left[\Gamma_{g}, \rho\right]$ ) denotes the vector space of all Siegel modular forms of weight $k$ (resp. of type $\rho$ ). The symbol " $:=$ " means that the expression on the right is the definition of that on the left. We denote by $\mathrm{Z}^{+}$the set of all positive integers. $F^{(k, l)}$ denotes the set of all $k \times l$ matrices with entries in a commutative ring $F$. For any $M \in F^{(k, l)},{ }^{t} M$ denotes the transpose matrix of $M$. For $A \in F^{(k, k)}, \sigma(A)$ denotes the trace of $A$. For $A \in F^{(k, l)}$ and $B \in F^{(k, k)}$, we set $B[A]={ }^{t} A B A . E_{g}$ denotes the identity matrix of degree $g$.

\section{SiNGULAR JACOBI FORMS}

In this section, we establish the notations and define the concept of singular Jacobi forms. Let

$$
\mathrm{Sp}(g, \mathbf{R})=\left\{\left.M \in \mathbf{R}^{(2 g, 2 g)}\right|^{t} M J_{g} M=J_{g}\right\}
$$

be the symplectic group of degree $g$, where

$$
J_{g}:=\left(\begin{array}{cc}
0 & E_{g} \\
-E_{g} & 0
\end{array}\right)
$$

It is easy to see that $\operatorname{Sp}(g, \mathbf{R})$ acts on $H_{g}$ transitively by

$$
M\langle Z\rangle:=(A Z+B)(C Z+D)^{-1},
$$

where $M=\left(\begin{array}{cc}A & B \\ C & D\end{array}\right) \in \operatorname{Sp}(g, \mathbf{R})$ and $Z \in H_{g}$. For two positive integers $g$ and $h$, we consider the Heisenberg group

$$
H_{\mathbf{R}}^{(g, h)}:=\left\{[(\lambda, \mu), \kappa] \mid \lambda, \mu \in \mathbf{R}^{(h, g)}, \kappa \in \mathbf{R}^{(h, h)}, \kappa+\mu^{t} \lambda \text { symmetric }\right\}
$$

endowed with the following multiplication law

$$
[(\lambda, \mu), \kappa] \circ\left[\left(\lambda^{\prime}, \mu^{\prime}\right), \kappa^{\prime}\right]:=\left[\left(\lambda+\lambda^{\prime}, \mu+\mu^{\prime}\right), \kappa+\kappa^{\prime}+\lambda^{t} \mu^{\prime}-\mu^{t} \lambda^{\prime}\right] .
$$


We define the semidirect product of $\operatorname{Sp}(g, \mathbf{R})$ and $H_{\mathbf{R}}^{(g, h)}$

$$
G^{J}:=\operatorname{Sp}(g, \mathbf{R}) \propto H_{\mathbf{R}}^{(g, h)}
$$

endowed with the following multiplication law

$$
\begin{aligned}
& (M,[(\lambda, \mu), \kappa]) \cdot\left(M^{\prime},\left[\left(\lambda^{\prime}, \mu^{\prime}\right), \kappa^{\prime}\right]\right) \\
& \quad:=\left(M M^{\prime},\left[\left(\tilde{\lambda}+\lambda^{\prime}, \tilde{\mu}+\mu^{\prime}\right), \kappa+\kappa^{\prime}+\tilde{\lambda}^{t} \mu^{\prime}-\tilde{\mu}^{t} \lambda^{\prime}\right]\right),
\end{aligned}
$$

with $M, M^{\prime} \in \operatorname{Sp}(g, \mathbf{R})$ and $(\tilde{\lambda}, \tilde{\mu}):=(\lambda, \mu) M^{\prime}$. It is easy to see that $G^{J}$ acts on $H_{g} \times \mathbf{C}^{(h, g)}$ transitively by

$$
(M,[(\lambda, \mu), \kappa]) \cdot(Z, W):=\left(M\langle Z\rangle,(W+\lambda Z+\mu)(C Z+D)^{-1}\right),
$$

where $M=\left(\begin{array}{ll}A & B \\ C & D\end{array}\right) \in \operatorname{Sp}(g, \mathbf{R}),[(\lambda, \mu), \kappa] \in H_{\mathbf{R}}^{(g, h)}$ and $(Z, W) \in H_{g} \times$ $\mathbf{C}^{(h, g)}$

Let $\rho$ be a rational representation of $G L(g, \mathbf{C})$ on a finite dimensional complex vector space $V_{\rho}$. Let $\mathscr{M} \in \mathbf{R}^{(h, h)}$ be a symmetric half-integral semipositive definite matrix of degree $h$. Let $C^{\infty}\left(H_{g} \times \mathbf{C}^{(h, g)}, V_{\rho}\right)$ be the algebra of all $C^{\infty}$ functions on $H_{g} \times \mathbf{C}^{(h, g)}$ with values in $V_{\rho}$. For $f \in C^{\infty}\left(H_{g} \times \mathbf{C}^{(h, g)}, V_{\rho}\right)$, we define

$$
\begin{aligned}
\left(\left.f\right|_{\rho, \mathscr{M}}\right. & {[(M,[(\lambda, \mu), \kappa])])(Z, W) } \\
:= & e^{-2 \pi i \sigma\left(\mathscr{M}[W+\lambda Z+\mu](C Z+D)^{-1} C\right)} \cdot e^{2 \pi i \sigma\left(\mathscr{M}\left(\lambda Z^{t} \lambda+2 \lambda^{t} W+\left(\kappa+\mu^{t} \lambda\right)\right)\right)} \\
& \times \rho(C Z+D)^{-1} f\left(M\langle Z\rangle,(W+\lambda Z+\mu)(C Z+D)^{-1}\right),
\end{aligned}
$$

where $M=\left(\begin{array}{ll}A & B \\ C & D\end{array}\right) \in \operatorname{Sp}(g, \mathbf{R})$ and $[(\lambda, \mu), \kappa] \in H_{\mathbf{R}}^{(g, h)}$.

Definition 2.1. Let $\rho$ and $\mathscr{M}$ be as above. Let

$$
H_{\mathbf{Z}}^{(g, h)}:=\left\{[(\lambda, \mu), \kappa] \in H_{\mathbf{R}}^{(g, h)} \mid \lambda, \mu \in \mathbf{Z}^{(h, g)}, \kappa \in \mathbf{Z}^{(h, h)}\right\} .
$$

A Jacobi form of index $\mathscr{M}$ with respect to $\rho$ on $\Gamma_{g}$ is a holomorphic function $f \in C^{\infty}\left(H_{g} \times \mathbf{C}^{(h, g)}, V_{\rho}\right)$ satisfying the following conditions (A) and (B):

(A) $\left.f\right|_{\rho, \mathscr{M}}[\tilde{\gamma}]=f$ for all $\tilde{\gamma} \in \Gamma_{g}^{J}:=\Gamma_{g} \propto H_{\mathrm{Z}}^{(g, h)}$.

(B) $f$ has a Fourier expansion of the following form:

$$
f(Z, W)=\sum_{\substack{T \geq 0 \\ \text { half-integral }}} \sum_{R \in Z^{(g, h)}} c(T, R) \cdot e^{2 \pi i \sigma(T Z)} \cdot e^{2 \pi i \sigma(R W)}
$$

with $c(T, R) \neq 0$ only if $\left(\begin{array}{cc}T & \frac{1}{2} R \\ \frac{1}{2} t R & \mathscr{M}\end{array}\right) \geq 0$.

If $g \geq 2$, the condition (B) is superfluous by the Koecher principle (cf. [Z], Lemma 1.6). We denote by $J_{\rho, \mathscr{M}}\left(\Gamma_{g}\right)$ the vector space of all Jacobi forms of index $\mathscr{M}$ with respect to $\rho$ on $\Gamma_{g}$. In the special case $V_{\rho}=\mathbf{C}, \rho(A)=$ $(\operatorname{det} A)^{k}(k \in \mathbf{Z}, A \in G L(g, \mathbf{C}))$, we write $J_{k, \mathscr{M}}\left(\Gamma_{g}\right)$ instead of $J_{\rho, \mathscr{M}}\left(\Gamma_{g}\right)$ and call $k$ the weight of a Jacobi form $f \in J_{k, \mathscr{M}}\left(\Gamma_{g}\right)$.

Ziegler (cf. [Z], Theorem 1.8 or [E-Z], Theorem 1.1) proves that the vector space $J_{\rho, \mathscr{M}}\left(\Gamma_{g}\right)$ is finite dimensional.

Definition 2.2. A Jacobi form $f \in J_{\rho, \mathscr{M}}\left(\Gamma_{g}\right)$ is said to be singular if it admits a Fourier expansion such that a Fourier coefficient $c(T, R)$ vanishes unless $\operatorname{det}\left(\begin{array}{cc}T & \frac{1}{2} R \\ \frac{1}{2} t R & \mathscr{M}\end{array}\right)=0$. 
Example 2.3. Let $S \in \mathbf{Z}^{(2 k, 2 k)}$ be a symmetric, positive definite, unimodular even integral matrix and $c \in \mathbf{Z}^{(2 k, h)}$. We define the theta series

$$
\vartheta_{S, c}^{(g)}(Z, W):=\sum_{\lambda \in \mathbf{Z}^{(2 k, g)}} e^{\pi\left\{\sigma\left(S \lambda Z^{t} \lambda\right)+2 \sigma\left({ }^{t} c S \lambda^{t} W\right)\right\}}, \quad Z \in H_{g}, W \in \mathbf{C}^{(h, g)} .
$$

We put $\mathscr{M}:=\frac{1}{2}{ }^{t} c S c$. We assume that $2 k<g+\operatorname{rank}(\mathscr{M})$. Then it is easy to see that $\vartheta_{S, c}^{(g)}$ is a singular Jacobi form in $J_{k, \mathscr{M}}\left(\Gamma_{g}\right)$ (cf. [Z], p. 212).

Remark 2.4. Without loss of generality, we may assume that $\mathscr{M}$ is a positive definite symmetric, half-integral matrix of degree $h$ (cf. [Z], Theorem 2.4). From now on, throughout this paper $\mathscr{M}$ is assumed to be positive definite.

\section{THE DIFFERENTIAL OPERATOR $M_{g, h, \mathscr{M}}$}

Let $\mathscr{P}_{g}$ be the open convex cone of positive definite matrices of degree $g$ in $\mathbf{R}^{g(g+1) / 2}$ defined in the introduction.

From now on, for $Y=\left(y_{\mu \nu}\right) \in \mathscr{P}_{g}$ and $V=\left(v_{k l}\right) \in \mathbf{R}^{(h, g)}$, we write

$$
\begin{aligned}
& d Y=\left(d y_{\mu \nu}\right), \quad d V=\left(d v_{k l}\right), \quad 1 \leq \mu, \nu \leq g, \quad 1 \leq k \leq h, \quad 1 \leq l \leq g, \\
& \frac{\partial}{\partial Y}=\left(\frac{1+\delta_{\mu \nu}}{2} \frac{\partial}{\partial y_{\mu \nu}}\right), \quad \frac{\partial}{\partial V}=\left(\frac{\partial}{\partial v_{k l}}\right) .
\end{aligned}
$$

For a real matrix $X$ of degree $g$ and an integer $k$ with $1 \leq k \leq g$, we denote by $C_{k}(X)$ the matrix of minors of degree $k$. We define the differential operator $M_{k, h}$ on $\mathscr{P}_{g} \times \mathbf{R}^{(h, g)}$ by

$$
M_{k, h}:=\sigma\left(C_{k}(Y) C_{k}\left(\frac{\partial}{\partial Y}+\frac{1}{8 \pi}^{t}\left(\frac{\partial}{\partial V}\right)\left(\frac{\partial}{\partial V}\right)\right)\right), \quad 1 \leq k \leq g .
$$

Following the notations of H. Maass (cf. [M], p. 67), the differential operator $M_{k, h}$ may be expressed as

$$
M_{k, h}=\sum_{\substack{1 \leq \alpha_{1}<\cdots<\alpha_{k} \leq g \\
1 \leq \beta_{1}<\cdots<\beta_{k} \leq g}}\left(\begin{array}{c}
\alpha_{1} \cdots \alpha_{k} \\
\beta_{1} \cdots \beta_{k}
\end{array}\right)_{Y} \cdot\left(\begin{array}{l}
\beta_{1} \cdots \beta_{k} \\
\alpha_{1} \cdots \alpha_{k}
\end{array}\right)_{\frac{\partial}{\partial Y}+\frac{1}{8 \pi}{ }^{\prime}\left(\frac{\partial}{\partial V}\right)\left(\frac{\partial}{\partial V}\right)}, \quad 1 \leq k \leq g .
$$

In particular, we are interested in the following differential operator

$$
M_{g, h}:=\operatorname{det}(Y) \cdot \operatorname{det}\left(\frac{\partial}{\partial Y}+\frac{1}{8 \pi}^{t}\left(\frac{\partial}{\partial V}\right)\left(\frac{\partial}{\partial V}\right)\right) .
$$

Lemma 3.1. Let $T={ }^{t} T \in \mathbf{R}^{(h, g)}$ and $R \in \mathbf{R}^{(g, h)}$. Then we have

$$
\frac{\partial}{\partial Y} e^{-2 \pi \sigma(T Y)}=-2 \pi e^{-2 \pi \sigma(T Y)} \cdot T
$$

and

$$
M_{g, h}\left(e^{-2 \pi \sigma(T Y+R V)}\right)=\left(-\frac{\pi}{2}\right)^{g} \operatorname{det}\left(Y \cdot\left(4 T-R^{t} R\right)\right) \cdot e^{-2 \pi \sigma(T Y+R V)} .
$$

Proof. (3.3) follows from an easy computation. We set

$$
P:=\left(P_{\mu \nu}\right):=\frac{\partial}{\partial Y}^{2}+\frac{1}{8 \pi}^{t}\left(\frac{\partial}{\partial V}\right)\left(\frac{\partial}{\partial V}\right) .
$$


Then we have

$$
P_{\mu \mu}:=\frac{\partial}{\partial y_{\mu \mu}}+\frac{1}{8 \pi} \sum_{k=1}^{h} \frac{\partial^{2}}{\partial v_{k \mu}^{2}}, \quad 1 \leq \mu \leq g
$$

and

$$
P_{\mu \nu}:=\frac{1}{2} \frac{\partial}{\partial y_{\mu \nu}}+\frac{1}{8 \pi} \sum_{k=1}^{h} \frac{\partial^{2}}{\partial v_{k \mu} \partial v_{k \nu}}, \quad 1 \leq \mu<\nu \leq g .
$$

We note that if $T=\left(t_{\mu \nu}\right), R=\left(r_{\mu k}\right)$ and $V=\left(v_{k \nu}\right)$, then

$$
\sigma(T Y)=\sum_{\mu=1}^{g} t_{\mu \mu} y_{\mu \mu}+2 \sum_{\mu<\nu} t_{\mu \nu} y_{\mu \nu}, \quad \sigma(R V)=\sum_{\mu=1}^{g} \sum_{k=1}^{h} r_{\mu k} v_{k \mu} .
$$

By an easy calculation, we get

$$
P_{\mu \nu}\left(e^{-2 \pi \sigma(T Y+R V)}\right)=-\frac{\pi}{2}\left(4 t_{\mu \nu}-\sum_{k=1}^{h} r_{\mu k} r_{\nu k}\right) \cdot e^{-2 \pi \sigma(T Y+R V)} .
$$

Thus we get

$$
\operatorname{det}(P)\left(e^{-2 \pi \sigma(T Y+R V)}\right)=\left(-\frac{\pi}{2}\right)^{g} \operatorname{det}\left(4 T-R^{t} R\right) \cdot e^{-2 \pi \sigma(T Y+R V)} .
$$

Consequently we obtain the desired result (3.4).

Now we let $\mathscr{M}$ be a symmetric positive definite, half-integral matrix of degree $h$. We define the differential operator $M_{g, h, \mathscr{M}}$ on $\mathscr{P}_{g} \times R^{(h, g)}$ by

$$
M_{g, h, \mathscr{M}}:=\operatorname{det}(Y) \cdot \operatorname{det}\left(\frac{\partial}{\partial Y}+\frac{1}{8 \pi}^{t}\left(\mathscr{M}^{-1 / 2} \frac{\partial}{\partial V}\right)\left(\mathscr{M}^{-1 / 2} \frac{\partial}{\partial V}\right)\right) .
$$

By changing the coordinate $V$ by $\widehat{V}=\mathscr{M}^{1 / 2} V$, we obtain $\partial / \partial \widehat{V}=\mathscr{M}^{-1 / 2} \partial / \partial V$. Thus (3.10) may be written as

$$
M_{g, h, \mathscr{M}}=\operatorname{det}(Y) \cdot \operatorname{det}\left(\frac{\partial}{\partial Y}+\frac{1}{8 \pi}^{t}\left(\frac{\partial}{\partial \widehat{V}}\right)\left(\frac{\partial}{\partial \widehat{V}}\right)\right) .
$$

Theorem 3.2. Let $T={ }^{t} T \in \mathbf{R}^{(h, g)}$ and $R \in \mathbf{R}^{(g, h)}$. Then we have

$$
\begin{aligned}
M_{g, h, \mathscr{M}}\left(e^{-2 \pi \sigma(T Y+R V)}\right) & \\
& =\left(-\frac{\pi}{2}\right)^{g} \operatorname{det}\left(Y \cdot\left(4 T-R \mathscr{K}^{-1 t} R\right)\right) \cdot e^{-2 \pi \sigma(T Y+R V)} .
\end{aligned}
$$

Proof. If we set $\widehat{R}=R \mathscr{M}^{-1 / 2}$, then $\widehat{R} \widehat{V}=R V$. Applying (3.11) to $e^{-2 \pi \sigma(T Y+R V)}$ $=e^{-2 \pi \sigma(T Y+\widehat{R} \widehat{V})}$ and using Lemma 3.1, we obtain the desired result (3.12).

\section{Proof of MAIN THEOREMS}

First we prove that a singular Jacobi form is characterized by the differential operator $M_{g, h, \mathscr{M}}$. 
Theorem 4.1. Let $f \in J_{\rho, \mathscr{M}}\left(\Gamma_{g}\right)$ be a Jacobi form of index $\mathscr{M}$ with respect to a rational representation $\rho$ of $G L(g, \mathbf{C})$. Then the following conditions are equivalent:

(1) $f$ is a singular Jacobi form.

(2) $f$ satisfies the differential equation $M_{g, h, \mathscr{M}} f=0$.

Proof. First we observe that for a Fourier coefficient $c(T, R)$ of $f(Z, W)$, we have

$$
\left(\begin{array}{cc}
T & \frac{1}{2} R \\
\frac{1}{2}^{t} R & \mathscr{M}
\end{array}\right)=\left(\begin{array}{cc}
E_{g} & \frac{1}{2} R \mathscr{M}^{-1} \\
0 & E_{h}
\end{array}\right)\left(\begin{array}{cc}
T-\frac{1}{4} R \mathscr{M}^{-1}{ }^{t} R & 0 \\
0 & \mathscr{M}
\end{array}\right)^{t}\left(\begin{array}{cc}
E_{g} & \frac{1}{2} R \mathscr{M}^{-1} \\
0 & E_{h}
\end{array}\right) .
$$

Thus it follows immediately that $\operatorname{det}\left(\begin{array}{c}T \frac{1}{2} \frac{1}{2}^{t} R \\ \mathscr{M}\end{array}\right)=0$ if and only if

$$
\operatorname{det}\left(4 T-R \mathscr{M}^{-1 t} R\right)=0 .
$$

Suppose $f \in J_{\rho, \mathscr{M}}\left(\Gamma_{g}\right)$ is singular. Then according to Theorem 3.2, we have

$$
\begin{aligned}
M_{g, h, \mathscr{M}} f(Z, W)= & \left(-\frac{\pi}{2}\right)^{g} \operatorname{det}(Y) \sum_{T, R} c(T, R) \operatorname{det}\left(4 T-R \mathscr{M}^{-1 t} R\right) \\
& \times e^{2 \pi i \sigma(T Z)} \cdot e^{2 \pi i \sigma(R W)} .
\end{aligned}
$$

Since $f$ singular, $c(T, R) \neq 0$ implies $\operatorname{det}\left(4 T-R \mathscr{M}^{-1}{ }^{t} R\right)=0$. Hence we obtain the equation $M_{g, h, \mathscr{M}} f=0$.

Conversely, we assume $M_{g, h, \mathscr{M}} f=0$. Then

$$
\begin{aligned}
& \left(-\frac{\pi}{2}\right)^{g} \operatorname{det}(Y) c(T, R) \operatorname{det}\left(4 T-R \mathscr{M}^{-1 t} R\right) \\
& \quad=\int_{0}^{1} \cdots \int_{0}^{1} M_{g, h, \mathscr{M}} f(Z, W) \cdot e^{-2 \pi i \sigma(T Z+R W)} d[X] d[U],
\end{aligned}
$$

where $Z=X+i Y, W=U+i V$ with real $X=\left(x_{\mu \nu}\right), Y=\left(y_{\mu \nu}\right), U=\left(u_{k l}\right)$, $V=\left(v_{k l}\right)$ and

$$
d[X] d[U]=d x_{11} d x_{12} \cdots d x_{g-1, g} d x_{g g} d u_{11} \cdots d u_{h, g-1} d u_{h g} .
$$

According to the assumption, we have for any $T$ and $R$

$$
c(T, R) \cdot \operatorname{det}\left(4 T-R \mathscr{M}^{-1}{ }^{t} R\right)=0 .
$$

This means that $c(T, R) \neq 0$ implies $\operatorname{det}\left(4 T-R \mathscr{M}^{-1}{ }^{t} R\right)=0$. Hence $f$ is singular.

Let $S$ be a symmetric positive definite integral matrix of degree $h$ and let $a, b \in \mathbf{Q}^{(h, g)}$. We consider

$$
\vartheta_{S, a, b}(Z, W):=\sum_{\lambda \in \mathbf{Z}^{(h, 8)}} e^{\pi i \sigma\left\{S\left((\lambda+a) Z^{t}(\lambda+a)+2(\lambda+a)^{t}(W+b)\right)\right\}}
$$

with characteristic $(a, b)$ converging uniformly on any compact subset of $H_{g} \times$ $\mathbf{C}^{(h, g)}$.

If $f$ is a Jacobi form in $J_{\rho, \mathscr{M}}\left(\Gamma_{g}\right)$, then according to [Z], we may write

$$
f(Z, W)=\sum_{a \in \mathcal{N}} f_{a}(Z) \cdot \vartheta_{2 \mathscr{M}, a, 0}(Z, W), \quad Z \in H_{g}, \quad W \in \mathbf{C}^{(h, g)},
$$

where $\mathscr{N}$ is a complete system of representatives of $(2 \mathscr{M})^{-1} \mathbf{Z}^{(h, g)} / \mathbf{Z}^{(h, g)}$ and $\left\{f_{a}: H_{g} \rightarrow V_{\rho} \mid a \in \mathscr{N}\right\}$ are uniquely determined holomorphic vector valued functions on $H_{g}$.

According to Yang (cf. [Y], Corollary 3.2), we have 
Proposition 4.2. Let $2 \mathscr{M}$ be unimodular. We assume that $\rho$ satisfies the following condition:

$$
\rho(A)=\rho(-A) \text { for all } A \in G L(g, \mathbf{C}) .
$$

Then we have

$$
J_{\rho, \mathscr{M}}\left(\Gamma_{g}\right)=\left[\Gamma_{g}, \tilde{\rho}\right] \cdot \vartheta_{2 \mathscr{M}, 0,0}(Z, W) \cong\left[\Gamma_{g}, \tilde{\rho}\right],
$$

where $\tilde{\rho}=\rho \otimes \operatorname{det}^{-h / 2}$.

Notation 4.3. In Propositoin 4.2, we denote the isomorphism of $J_{\rho, \mathscr{M}}\left(\Gamma_{g}\right)$ onto $\left[\Gamma_{g}, \rho \otimes \operatorname{det}^{-h / 2}\right]$ by

$$
S_{\rho, \mathscr{M}}: J_{\rho, \mathscr{M}}\left(\Gamma_{g}\right) \rightarrow\left[\Gamma_{g}, \rho \otimes \operatorname{det}^{-h / 2}\right] .
$$

Definition 4.4. An irreducible finite dimensional representation $\rho$ of $G L(g, \mathbf{c})$ is determined uniquely by its highest weight $\left(\lambda_{1}, \lambda_{2}, \ldots, \lambda_{g}\right) \in \mathbf{Z}^{g}$ with $\lambda_{1} \geq$ $\lambda_{2} \geq \cdots \geq \lambda_{g}$. We denote this representation by $\rho=\left(\lambda_{1}, \lambda_{2}, \ldots, \lambda_{g}\right)$. The number $k(\rho):=\lambda_{g}$ is called the weight of $\rho$.

Theorem 4.5. Let $2 \mathscr{M}$ be a symmetric, positive definite, unimodular even matrix of degree $h$. Assume that $\rho$ is irreducible and satisfies the condition (4.3). Then a nonvanishing Jacobi form in $J_{\rho, \mathscr{M}}\left(\Gamma_{g}\right)$ is singular if and only if $2 k(\rho)<g+h$.

Proof. According to Proposition 4.2, we have

$$
J_{\rho, \mathscr{K}}\left(\Gamma_{g}\right)=\left[\Gamma_{g}, \rho \otimes \operatorname{det}^{-h / 2}\right] \cdot \vartheta_{2 \mathscr{M}, 0,0}(Z, W) .
$$

For any $f \in J_{\rho, \mathscr{M}}\left(\Gamma_{g}\right), f=S_{\rho, \mathscr{M}}(f) \cdot \vartheta_{2 \mathscr{M}, 0,0}(Z, W)$. First of all, we observe that the Fourier coefficients $b(T, R)$ of $\vartheta_{2 M, 0,0}(Z, W)$ is given by

$$
b(T, R)= \begin{cases}1 & \text { if }{ }^{\exists} \lambda \in \mathbf{Z}^{(h, g)} \text { s.t. } T=\mathscr{M}[\lambda],{ }^{t} R=2 \mathscr{M} \lambda, \\ 0 & \text { otherwise. }\end{cases}
$$

Obviously we have $4 T-R \mathscr{M}^{-1}{ }^{t} R=0$ for $T, R$ with $b(T, R) \neq 0$. Let $a(T)$ and $c(T, R)$ be the Fourier coefficients of $S_{\rho, \mathscr{M}}(f)(Z)$ and $f(Z, W)$ respectively. If $c(T, R) \neq 0$, then $c(T, R)=a\left(T_{1}\right) b\left(T_{2}, R\right)$ with $T=T_{1}+T_{2}$ because $T_{2}$ is uniquely determined by $R$.

Now we suppose that $f(Z, W) \neq 0$ is singular. If $a\left(T_{1}\right) \neq 0$ for some half integral $T_{1} \geq 0$, then there exist $T_{2} \geq 0$ and $R \in \mathbf{Z}^{(g, h)}$ such that $b\left(T_{2}, R\right) \neq 0$ and hence $c\left(T_{1}+T_{2}, R\right)=a\left(T_{1}\right) b\left(T_{2}, R\right) \neq 0$ is the Fourier coefficient of $f(Z, W)$. By assumption and the fact that $4 T_{2}-R \mathscr{M}^{-1} t R=0$, we have

$$
\operatorname{det}\left(4\left(T_{1}+T_{2}\right)-R \mathscr{M}^{-1}{ }^{t} R\right)=\operatorname{det}\left(4 T_{1}\right)=0 .
$$

Hence $S_{\rho, \mathscr{M}}(f) \neq 0$ is singular. According to [W], Satz 4, we obtain the condition $2 k(\rho)<g+h$. Conversely, suppose $2 k(\rho)<g+h$. Then, according to [W], Satz 4, $S_{\rho, \mathscr{M}}(f)$ is singular. If $c(T, R) \neq 0$, then we have $c(T, R)=$ $a\left(T_{1}\right) b\left(T_{2}, R\right)$ for uniquely determined half-integral $T_{1}$ and $T_{2}$ with $T=$ $T_{1}+T_{2}$. Since $a\left(T_{1}\right) \neq 0$ and $S_{\rho, \mathscr{M}}(f)$ is singular, $\operatorname{det}\left(T_{1}\right)=0$. Using the fact that $4 T_{2}-R \mathscr{M}^{-1}{ }^{t} R=0$, we obtain

$$
\operatorname{det}\left(4 T-R \mathscr{M}^{-1}{ }^{1} R\right)=\operatorname{det}\left(4 T_{1}\right)=0 .
$$

Hence $f(Z, W)$ is singular. This completes the proof. 
Remark 4.6. For general $\rho$ and $\mathscr{M}$ without the above assumptions on them, it is possible to prove that a nonvanishing Jacobi form $f \in J_{\rho, \mathscr{M}}\left(\Gamma_{g}\right)$ is singular if and only if $2 k(\rho)<g+h$ since [W], Satz 4 also holds for normal subgroups of finite index in $\Gamma_{g}$.

Remark 4.7. Ziegler (cf. [Z], Theorem 3.12) proved that a strongly singular Jacobi form may be written as a linear combination of theta series $\vartheta_{S, c}^{(g)}$ (cf. (2.3)).

Finally we prove

Theorem 4.8. Let $\mathscr{M}$ be a symmetric, positive definite half-integral matrix of degree $h$. Then for all $a, b \in \mathbf{Q}^{(h, g)}$, the theta series $\vartheta_{2 /, a, b}(Z, W)$ satisfies the differential equation

$$
M_{g, h, \mathscr{M}} \vartheta_{2 \mathscr{M}, a, b}(Z, W)=0 .
$$

Proof. For each $\lambda \in \mathbf{Z}^{(h, g)}$, we put

$$
T_{\lambda}:={ }^{t}(\lambda+a) \mathscr{M}(\lambda+a), \quad R_{\lambda}:=2^{t}(\lambda+a) \mathscr{M} .
$$

According to Theorem 3.2, we have

$$
\begin{aligned}
M_{g, h, \mathscr{M}} \vartheta_{2 \mathscr{M}, a, b}(Z, W) & \\
= & \left(-\frac{\pi}{2}\right)^{g} \operatorname{det}(Y) \cdot \sum_{\lambda \in \mathbf{Z}^{(h, g)}} \operatorname{det}\left(4 T_{\lambda}-R_{\lambda} \mathscr{M}^{-1}{ }^{t} R_{\lambda}\right) \\
& \cdot e^{\left.2 \pi i \sigma\left\{\mathscr{M}\left((\lambda+a) Z^{t}(\lambda+a)+2(\lambda+a)\right)^{t}(W+b)\right)\right\}} .
\end{aligned}
$$

It is easy to show that $\operatorname{det}\left(4 T_{\lambda}-R_{\lambda} \mathscr{M}^{-1}{ }^{t} R_{\lambda}\right)=0$ for all $\lambda \in \mathbf{Z}^{(h, g)}$. Hence we obtain the equation (4.5).

\section{EIGENFUNCTIONS OF $M_{g, h, \mathscr{M}}$}

In this section, we give eigenfunctions of the differential operator $M_{g, h, \mathscr{M}}$. For $Y \in \mathscr{P}_{g}$, we let $Y=T[Q]$ be the Jacobian decomposition of $Y$ such that

$$
T=\left(\begin{array}{cccc}
t_{1} & 0 & \ldots & 0 \\
0 & t_{2} & \ldots & 0 \\
\vdots & \vdots & \ddots & \vdots \\
0 & 0 & \ldots & t_{g}
\end{array}\right), \quad Q=\left(\begin{array}{cccc}
1 & * & \ldots & * \\
0 & 1 & \ldots & * \\
\vdots & \vdots & \ddots & \vdots \\
0 & 0 & \ldots & 1
\end{array}\right),
$$

where $T$ is a diagonal matrix with all $t_{\nu}>0(\nu=1, \ldots, g)$ and $Q$ is an upper triangular matrix with ones in the main diagonal (cf. [M]). We call the $t_{\nu}$ $(1 \leq \nu \leq g)$ and the elements $q_{k l}(1 \leq k<l \leq g)$ of $Q$ Jacobian coordinates of $Y$.

For $s=\left(s_{1}, \ldots, s_{g}\right) \in \mathbf{C}^{g}$, we define the function $f_{s}(Y)$ on $\mathscr{P}_{g}$ by

$$
f_{s}(Y):=\prod_{k=1}^{g} t_{k}^{s_{k}+k / 2-(g+1) / 4}, \quad Y=T[Q] \in \mathscr{P}_{g} .
$$

We put

$$
\varepsilon:=\left(\varepsilon_{11}, \ldots, \varepsilon_{1 g}, \ldots, \varepsilon_{h 1}, \ldots, \varepsilon_{h g}\right) \in \mathbf{Z}_{2}^{h g}, \quad \mathbf{Z}_{2}=\{0,1\} .
$$

That is, $\varepsilon_{i j}=0$ or 1 for $1 \leq i \leq h$ and $1 \leq j \leq g$. 
Theorem 5.1. Let $\mathscr{M}$ be a half-integral positive definite symmetric matrix of degree $h$. Let $\mathscr{M}^{1 / 2}$ be the unique positive definite symmetric matrix such that $\left(\mathscr{M}^{1 / 2}\right)^{2}=\mathscr{M}$. We put $\mathscr{M}^{1 / 2}:=\left(\alpha_{i j}\right), 1 \leq i, j \leq h$. Then for each $s=\left(s_{1}, \ldots, s_{g}\right) \in \mathbf{C}^{g}$ and $\varepsilon=\left(\varepsilon_{11}, \ldots, \varepsilon_{h 1}, \ldots, \varepsilon_{h g}\right) \in \mathbf{Z}_{2}^{h g}$, the function $f_{s, \varepsilon, \mathscr{M}}(Y, V)$ on $\mathscr{P}_{g} \times \mathbf{R}^{(h, g)}$ defined by

$$
f_{s, \ell, \mathscr{M}}(Y, V):=f_{s}(Y) \cdot\left(\sum_{k=1}^{h} \alpha_{1 k} v_{k 1}\right)^{\varepsilon_{11}} \ldots\left(\sum_{k=1}^{h} \alpha_{i k} v_{k j}\right)^{\varepsilon_{i j}} \ldots\left(\sum_{k=1}^{h} \alpha_{h k} v_{k g}\right)^{\varepsilon_{h g}}
$$

is an eigenfunction of the differential operator $M_{g, h, \ldots}$ with the eigenvalue $\lambda_{s, \ell, \mathscr{N}}=\prod_{k=1}^{g}\left(s_{k}+(g-1) / 4\right)$.

Proof. We leave the proof to the interested reader.

\section{ACKNOWLEDGEMENTS}

I would like to thank D. Zagier and W. Kohnen for many useful discussions and their interest in this problem. This work was done in part during my stay at the Max-Planck-Institut für Mathematik. I am very grateful to the institute for the hospitality and support.

\section{REFERENCES}

[E-Z] M. Eichler and D. Zagier, The theory of Jacobi forms, Progress in Math., 55, Birkhäuser, Boston, Basel, and Stuttgart, 1985.

[M] H. Maass, Siegel modular forms and Dirichlet series, Lecture Notes in Math., vol. 216, Springer-Verlag, Berlin and New York, 1971.

[W] R. Weissauer, Vektorwertige Siegelsche Modulformen kleinen Gewichtes, J. Reine Angew. Math. 343 (1983), 184-202.

[Y] J.-H. Yang, The Siegel-Jacobi operator, Abh. Math. Sem. Univ. Hamburg 63 (1993), 135-146.

[Z] C. Ziegler, Jacobi forms of higher degree, Abh. Math. Sem. Univ. Hamburg 59 (1989), 191-224.

Department of Mathematics, Inha University, Incheon 402-751, Republic of Korea

E-mail address: jhyang@munhak. inha.ac.kr 